\title{
Unknown Surface Modeling Method Based on High-precision CMM
}

\author{
Yue Ping Chen*, Yi Chang Lu \\ Email address: \\ chenyueping99@126.com (Yue Ping Chen), 1014170898@qq.com(Yi Chang Lu) \\ ${ }^{*}$ Corresponding author
}

School of Mechanical and Transportation, Guangxi University of Science and Technology, Liuzhou, China

\section{To cite this article:}

Yue Ping Chen, Yi Chang Lu. Unknown Surface Modeling Method Based on High-precision CMM. International Journal of Mechanical Engineering and Applications. Vol. 9, No. 1, 2021, pp. 1-5. doi: 10.11648/j.ijmea.20210901.11

Received: December 30, 2020; Accepted: January 12, 2021; Published: January 22, 2021

\begin{abstract}
Reverse engineering is typically applied to solve the CAD model of unknown parts. As modern industry requirements on the accuracy of complex surfaces increase, setting up the high-precision CAD modeling of unknown complex surface parts through reverse engineering becomes an interesting research topic. Here, we present a method that combines manual measurement and automatic measurement with a high-precision coordinate measuring machine (CMM) to measure unknown complex-surface to obtain CAD model. In this study, the unknown complex-surface was fixed on the CMM worktable, and cancelling the probe compensation function of PC-DMIS before measurement. Then, 490 measuring points were obtained by manually moving the probe with a diameter of $5 \mathrm{~mm}$ to measure surface. The measured points generated the CAD model, the CAD model was offset a probe radius, and determined the rough contours of the surface. This CAD model was automatically measured in a CMM, and the function of the "automatic measurement model" was iterated to obtain a high-accuracy CAD model. Each measurement was arranged with $20 \times 20$ measuring points. The accuracy of the CAD model obtained by each iterative measurement would be improved, and finally a high-precision CAD model was obtained. After 7 iterations, the final accuracy of the unknown surface CAD model was as high as $0.004 \mathrm{~mm}$ as compared to that of the actual part.
\end{abstract}

Keywords: Reverse Engineering, Coordinate Measuring Machine, Complex Surface, Iteration

\section{Introduction}

Through numerous explorations and experiments, many methods and instruments have been applied to reverse engineer model parts [1]. There are two ways of measuring and modeling unknown surfaces, namely touch-trigger probe and non-contact measurement. With the improvement of the complexity and the accuracy of part measurement, there are some measurement methods that combine the two. However, the non-contact measurement accuracy such as that of laser scanning is insufficient, and the output of the point cloud data is huge [2], which requires special software for post-processing; therefore, this method is not suitable for unknown high- precision model reconstruction of a curved surface. From the touch-trigger probe perspective, the efficiency of modeling by manual measurement is low, and the accuracy of modeling by pure automatic measurement is low. Moreover, people focus a lot on non-contact aspects.
Therefore, there has been little research on the reverse modeling of unknown surfaces using a high-precision CMM combined with manual and automatic measurement methods.

Several studies have been conducted on the modeling of parts with the knowledge of reverse engineering, particularly from the non-contact aspect. Some researchers have focused on the study of the 3D point cloud algorithm [3-7]. Numerous scholars have attempted to combine the tactile probe and the non-contact multi-probe measurement methods. A tactile probe measures the key features of the parts to compensate for the data of the laser line scanner, to carry out precise geometric feature reverse engineering [2, 8, 9]. Other researchers have discussed the use of shape reconstruction to improve the performance of parts [10-12]. These studies were based on non-contact measurement. However, the accuracy of non-contact measurements, such as laser scanning, is heavily dependent on the material, and there are many influencing factors [2]. Song et al. proposed a method 
to reconstruct the edge surface with a laser scanning confocal microscope, but the equipment used in this method is special and expensive [13]. Therefore, there have been few studies on the reverse modeling of unknown surfaces using the common CMM combined with manual and automatic measurement methods.

In this paper, we present a method of modeling unknown surfaces using a CMM combined with manual and automatic measurement methods. The function of the "automatic measurement model" is used to improve the accuracy of modeling and determine the CAD model of the unknown surfaces.

\section{Measurement Method}

The CAD modeling of an unknown surface does not provide a digital model, and thus, the surface cannot be measured automatically using a CMM. The rough profile of the unknown complex surface can be determined by manual measurement and corrected by the offset function. The offset surface is measured and modeled by automatic measurement, and the deviation between the current measurement data and the reference CAD surface is judged until the average deviation of the measurement data is less than the preset value.

The modeling method of an unknown complex surface is as follows.

Step 1. Turn off the probe radius compensation. The unknown complex surface is measured manually by the $\mathrm{CMM}$ to obtain measurement data $\mathrm{A}$. The software generates CAD surface A from data A and then offsets surface A along the normal vector direction of the surface according to the probe radius parameter. The offset formula is as follows:

$$
P_{d}=P \pm d \cdot N(x, y, z)
$$

where $P$ is the surface obtained by manual measurement, $P_{d}$ is an equidistant surface with a distance of $\mathrm{d}$, and $N(x, y, z)$ is the unit normal vector at the corresponding $(x, y, z)$ position, as shown in Figure 1.

Step 2. Turn on the probe radius compensation. The grid method is used to generate equidistant measurement points on the offset surface, and the CMM is used to measure the measurement points automatically; measurement data B is thus obtained.

Step 3. Use measurement data B as the reference data file, and generate the reference CAD surface using the software. The reference CAD surface is used to generate the measurement points by the grid method, and measurement data $\mathrm{C}$ is obtained by CMM measurement.

Step 4. Calculate the average distance deviation between measurement data $\mathrm{C}$ and the reference $\mathrm{CAD}$ surface generated in Step 3 as follows:

$$
\begin{gathered}
\varepsilon=\left(\sum_{j=1}^{n} \alpha_{j}\right) / n \\
\alpha=\sqrt{\left(x_{s}-x_{i}\right)^{2}+\left(y_{s}-y_{i}\right)^{2}+\left(z_{s}-z_{i}\right)^{2}}
\end{gathered}
$$

where $n$ is the total number of measurement points, $j$ is the variable representing the number of measurement points, $\left(x_{i}\right.$, $\left.y_{i}, z_{i}\right)$ is the coordinate of a measurement point in measurement data $\mathrm{C},\left(x_{s}, y_{s}, z_{s}\right)$ is the nearest coordinate from the reference CAD surface to the measurement point $\left(x_{i}, y_{i}\right.$, $z_{i}$ ), and $\alpha$ is the distance deviation between the coordinate of a measurement point in measurement data $\mathrm{C}$ and the reference CAD surface.

If the average distance deviation is less than the preset error value, data $\mathrm{C}$ is used to generate the $\mathrm{CAD}$ surface through software as the final modeling result; if the average distance deviation is greater than the preset error value, replace data $B$ with data $C$ as the reference data, and repeat Steps 3 and 4 . The flowchart of the proposed methodology is illustrated in Figure 2, where $\varepsilon_{\mathrm{TH}}$ is the threshold.

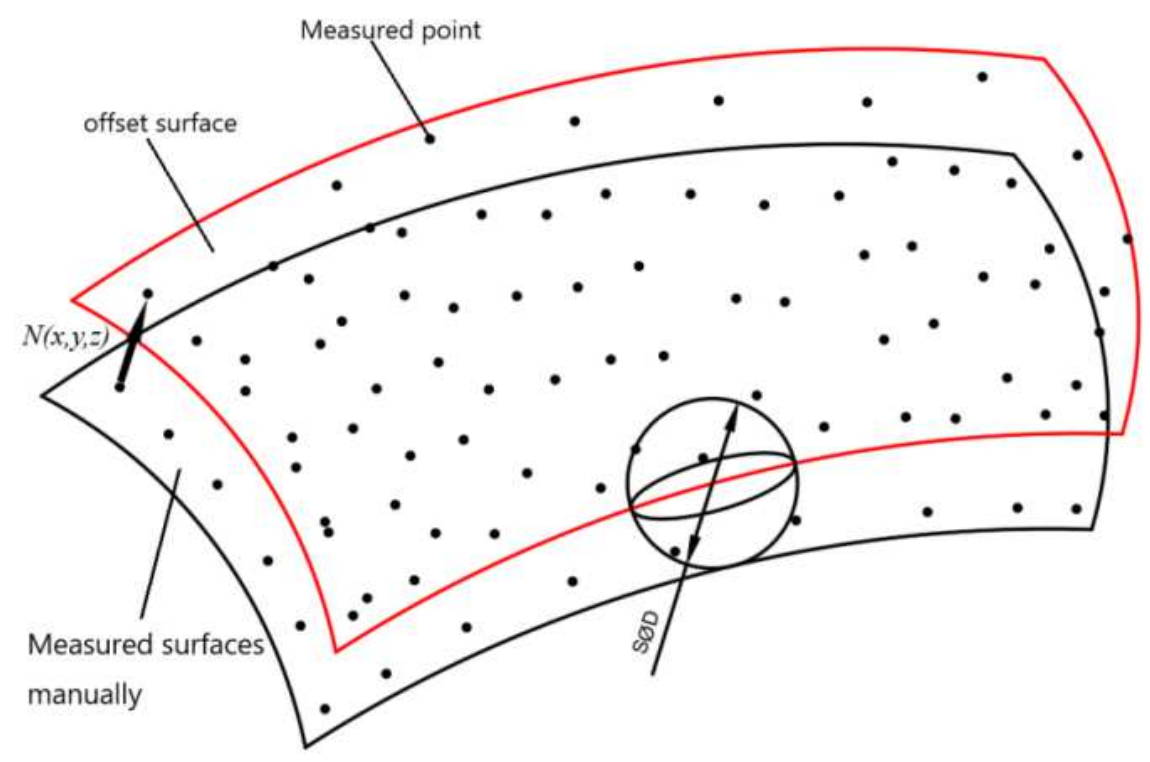

Figure 1. Offset Surface from measured surface. 


\section{Experiment}

In this study, an experiment of an unknown complex surface measurement was carried out using a bridge CMM of HEXAGON, as shown in Figure 3. The measurement software used was PC-DMIS, and accuracy of the machine was $\mathrm{MPEE}=0.9+\mathrm{L} / 400 \mu \mathrm{m}$. The $\mathrm{CMM}$ was equipped with a contact trigger probe, and the diameter of the ruby probe was $5 \mathrm{~mm}$. The part to be tested was a complex surface; it measured $110 \mathrm{~mm} \times 110 \mathrm{~mm} \times 30 \mathrm{~mm}$ and was made of an aluminum alloy, as shown in Figure 4.

We manually measured the surface, turned off the probe radius compensation in the settings, and set the speed of contacting the measured object to as slow as possible. In all, 490 measurement points were obtained by manually measuring the surface, as shown in Figure 5. The surface measurement points obtained by the manual measurement were fitted to generate the surface. During the manual measurement, the probe radius compensation setting of the CMM was turned off, so there was a probe radius error on the generated surface, and therefore, it was necessary to correct the surface by $2.5 \mathrm{~mm}$ to obtain offset surface A, as shown in Figure 6.

We arranged $20 \times 20$ measurement points on surface A, generated the CAD model, and imported it into the CMM. Then, we turned on the probe radius compensation, established an automatic measuring coordinate system, measured the curved surface to obtain the measurement point data, and generated surface B. Next, we arranged $20 \times 20$ measurement points on surface $\mathrm{B}$ and used the measurement point data obtained by the CMM to fit the next surface. We used the "automatic measurement model" function of the measurement software PC-DMIS in the inspection for the iteration.
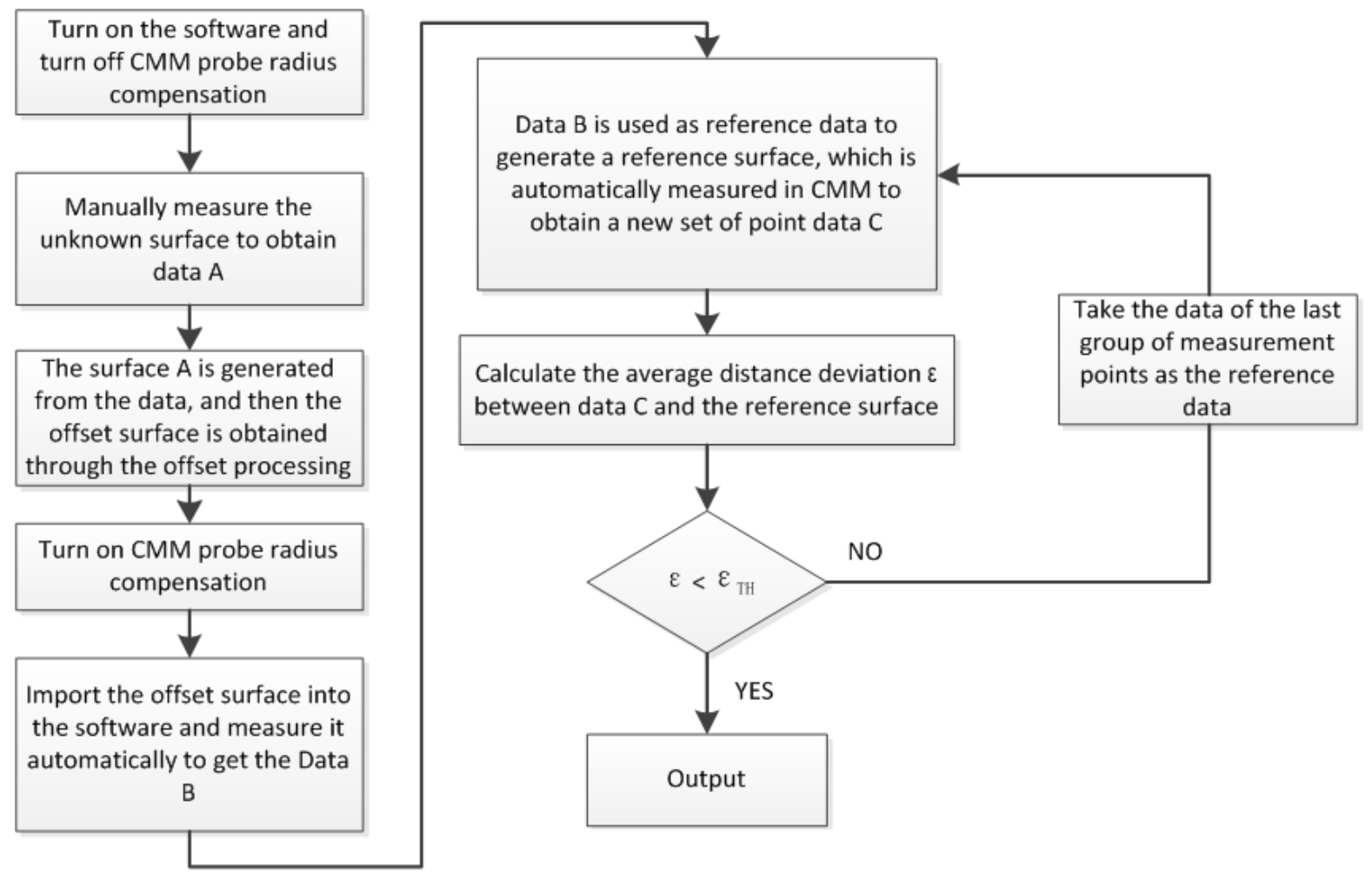

Figure 2. Flowchart of the proposed methodology.

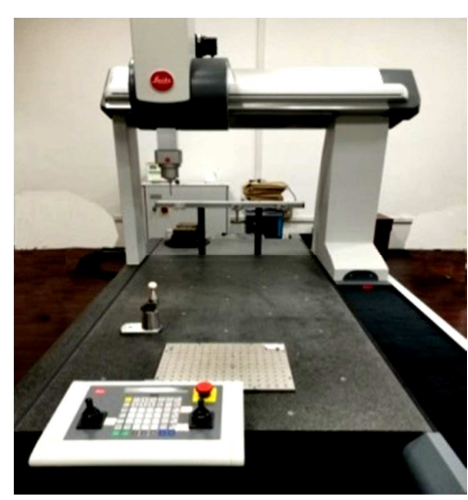

Figure 3. Experiment instruments.

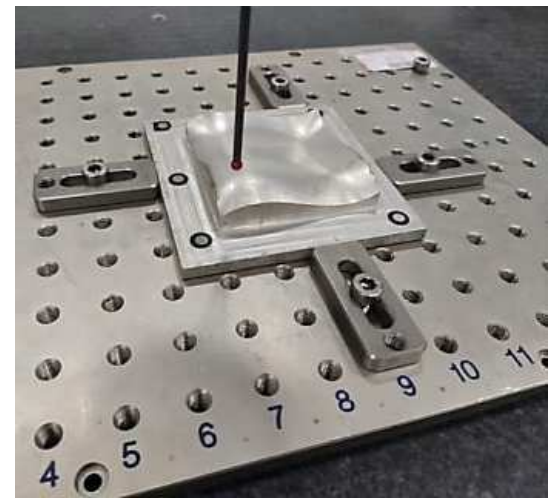

Figure 4. Unknown surface part used in the experiment. 


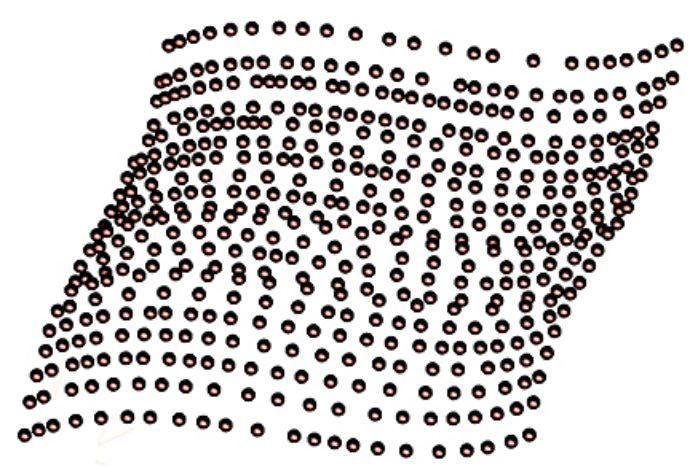

Figure 5. Points obtained by manual measurement.

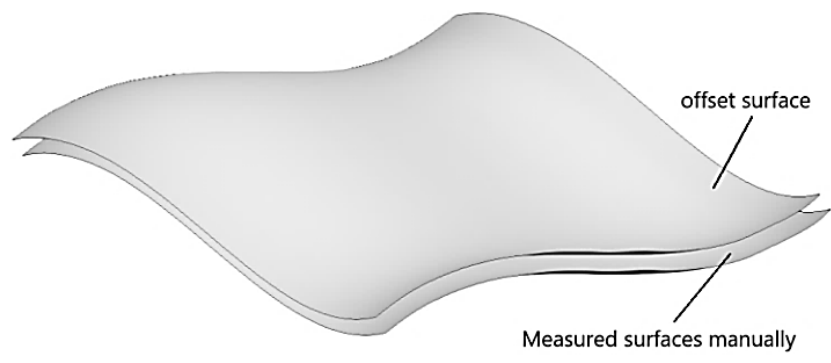

Figure 6. Manual measurement of fitted offset surface.

\section{Discussion and Results}

Generally, the uncertainty of optical scanning measurement is between $0.02-0.1 \mathrm{~mm}$, and contact measurement is between $0.001-0.005 \mathrm{~mm}[14]$. When the parts are complex, the reverse precision is required to be high, and laser scanning is easily affected by external factors in practice, the measurement accuracy is low [15], so the contact measurement is usually chosen for reverse. Moreover, the contact measurement can be planned manually, so that more measurements can be obtained in the area with large or sharp curvature change. Contact measurement can plan the area of the measured object manually, and measure the feature line and curve grid which play a key role in the shape of the object [16]. In reference [17], in order to solve the problem that complex multi-curved surface had many dead angles and the scanning and optical measurement methods could hardly be realized, the author manually measured parts in coordinate measuring machine. And the author mentioned that the low efficiency of manual mode, the function of automatic tracking measurement of unknown curve and surface of measurement software couldn't be used and the compensation of probe radius were still the problems that need to be paid attention to improve the efficiency and accuracy of the measurement.

So, before using the high-precision CMM to measure the unknown surface manually, we turned off the CMM probe radius compensation setting, because a manual measurement surface cannot guarantee that the measurement direction of the probe will be the normal direction of the point to be measured on the surface. Furthermore, in the case of manual measurement, the same speed and strength of measurement cannot be maintained. Therefore, the manual measurement of unknown surfaces by the CMM contained a probe radius error and other manual measurement errors. The CMM probe used ruby with high wear resistance and hardness, and the CMM was equipped with a reference sphere to verify the probing accuracy. Therefore, the effect of the CMM probe wear on the measurement could be ignored. The manual measurement performed to obtain the surface could eliminate the probe radius error by offsetting one probe radius. In the case of the automatic measurement of the CMM, the same speed and strength of measurement can be maintained; therefore, the automatic measurement of surfaces by the CMM can avoid the manual measurement errors. The offset surface was measured automatically using an iterative method, which could make the surface obtained by the CMM automatic measurement approach the actual part surface gradually. Table 1 displays the average deviations of the surface corresponding to the number of surface iterations. With an increase in the number of iterations, the mean distance deviations and variances of the surface decreased gradually, as shown in Figure 7. According to the mean distance deviation, the accuracy was as high as $0.004 \mathrm{~mm}$ after seven iterations.

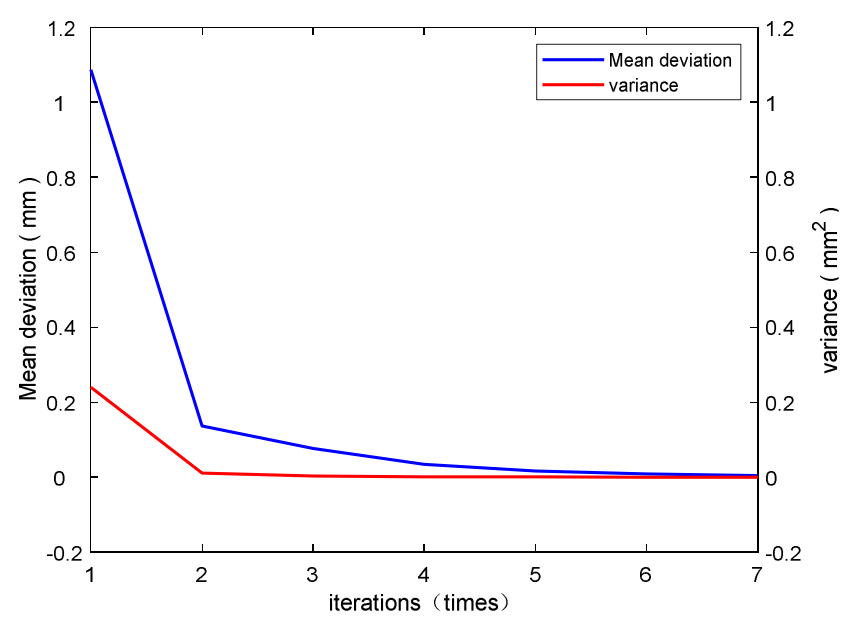

Figure 7. Trend of surface accuracy after iteration.

Table 1. Average deviation values and variances of the surface corresponding to the number of surface iterations.

\begin{tabular}{lllllll}
\hline Iteration times & $\mathbf{1}$ & $\mathbf{2}$ & $\mathbf{3}$ & $\mathbf{4}$ & $\mathbf{5}$ & $\mathbf{6}$ \\
\hline Surface mean deviation $(\mathrm{mm})$ & 1.0873 & 0.1368 & 0.0764 & 0.034 & 0.0159 & 0.0080 \\
Variance (mm2) & 0.2399 & 0.0112 & 0.0027 & 0.00054 & 0.00015 & 0.00004 \\
\hline
\end{tabular}

\section{Conclusions}

The method of establishing high-precision model of unknown surface was proposed in the present work. The main conclusions can be summarized as follows:

1. A method combines manual measurement and automatic measurement was proposed to establish the unknown complex surface model.

2. Manual measurement was used to determine the rough 
contours of the unknown surface and generate the initial CAD model of the surface. Automatic measurement could further improved the accuracy of the surface.

3. The CAD model of initial surface was imported into CMM for automatic measurement, and the function of "automatic measurement model" was used for iterative measurement. The final $\mathrm{CAD}$ model accuracy was $0.004 \mathrm{~mm}$.

\section{Acknowledgements}

This work was financially supported by the National Nature Science Foundation of China (51565006), the Natural Science Foundation of Guangxi Province (2018GXNSFAA050085, 2014GXNSFAA118337), the Science Research Innovation Team Project of Guangxi Provincial Education Department, and the Science Research Innovation Team Project of Guangxi University of Science and Technology. We thank Let Pub (www.letpub.com) for its linguistic assistance during the preparation of this manuscript.

\section{References}

[1] M. Berger, A. Tagliasacchi. (2017). A Survey of Surface Reconstruction from Point Clouds. Computer Graphics Forum, 36 (1): 301-329.

[2] J. Herráez, J. C. Martínez. (2016). 3D modeling by means of videogrammetry and laser scanners for reverse engineering. Measurement, 87: 216-227.

[3] Zhu, Kukko. (2019). Multisource Point Clouds, Point Simplification and Surface Reconstruction. Remote Sensing, 11 (2): 2659.

[4] Y. Yi, F. Hairong. (2020). Three-dimensional point cloud data subtle feature extraction algorithm for laser scanning measurement of large-scale irregular surface in reverse engineering. Measurement, 151: 107220.

[5] L. C. Jin, W. G. Wan. (2013). Globally optimal estimate for variational surface reconstruction. The Imaging Science Journal, 60 (2): 97-102.
[6] Maiti, D. Chakravarty. (2016). Performance analysis of different surface reconstruction algorithms for 3D reconstruction of outdoor objects from their digital images. Springerplus, 5 (1): 932.

[7] L. Cao, F. J. Verbeek. (2013). Analytical evaluation of algorithms for point cloud surface reconstruction using shape features. Journal of Electronic Imaging, 22 (4): 043008.

[8] L. Feng, A. P. Longstaff. (2014). Rapid and accurate reverse engineering of geometry based on a multi-sensor system. The International Journal of Advanced Manufacturing Technology, 74 (1-4): 369-382.

[9] X. Zexiao, W. Jianguo. (2005). Complete 3D measurement in reverse engineering using a multi-probe system. International Journal of Machine Tools and Manufacture, 45: (12-13) 1474-1486.

[10] N. Anwer, L. Mathieu. (2016). From reverse engineering to shape engineering in mechanical design. CIRP Annals, 65 (1): $165-168$.

[11] Ciocănea, S. Nicolaie. (2017). Reverse Engineering for the Rotor Blades of a Horizontal Axis Micro-hydrokinetic Turbine. Energy Procedia, 112: 35-42.

[12] A. P. Valerga, M. Batista. (2015). Reverse Engineering Based Methodology for Modelling Cutting Tools. Procedia Engineering, 132: 1144-1151.

[13] S. Shuquan, L. Bin. (2020). A new cutting edge surface reconstruction method for hard whirling tools. Measurement, 151: 107276.

[14] Mu Lu-xi. (2012). Study on Key Technologies of Complex Surfaces on Machine Measurement and Application [D]. Huazhong University of Science and Technology.

[15] Igor B, Nick VG. (2011). Accuracy improvement of laser line scanning for feature measurements on CMM [J]. Optics and Lasers in Engineering, 49 (11).

[16] ZHANG Li-yan. (2002). Model Reconstruction Based on Artificial-Programmed Coordinate Measuring [J]. Mechanical Science and Technology for Aerospace Engineering, 328-330.

[17] WANG Li-xin. (2008). Reverse Engineeing CAD Moldeling for Complex Multi-surface Based on CMM Measuring [J]. Machine Tool \& Hydraulics, 227-230. 